\title{
Assessment of the Elastic Component in Tunica Adventitia of Some Arteries in Lamb
}

\author{
Dalma CSIBI ${ }^{1}$, Flavia RUXANDA ${ }^{1}$, Vasile RUS ${ }^{1}$, Cristian MARTONOS ${ }^{1}$, Cristian RAŢIU ${ }^{2}$, Viorel MICLĂUŞ ${ }^{*}$, \\ Aurel DAMIAN $^{1}$ \\ ${ }^{1}$ University of Agricultural Sciences and Veterinary Medicine Cluj-Napoca, Romania. \\ ${ }^{2}$ University of Oradea, Romania. \\ *Corresponding author: vmiclaus@usamvcluj.ro
}

Bulletin UASVM Veterinary Medicine 72(2) / 2015,

Print ISSN 1843-5270; Electronic ISSN 1843-5378

DOI:10.15835/buasvmcn-vm: 11380

\begin{abstract}
Tunica adventitia of large caliber vessels in lambs presents a highly different thickness from one segment to another, depending on its position in relation to the heart and the irrigated area. In the case of all the studied arteries, adventitia is formed out of collagen and elastic fibers. In some arteries, adventitia contains a small quantity of elastic fibers (ascending aorta, aortic arch, thoracic descending aorta, bicarotid trunk, left subclavian artery). Other arteries contain elastic fibers in a markedly larger quantity, without exceeding a certain limit (abdominal descending aorta, left and right internal iliac arteries, brachiocephalic trunk, right subclavian artery). There are also vessels in which the elastic component from tunica adventitia is formed out of thick and numerous fibers, so that the elastic component in adventitia markedly exceeds the one from tunica media (left and right external iliac arteries, left and right carotid arteries). The large quantity of elastic tissue in tunica adventitia of these vessels seems to be an adaptation to the external solicitations that exert upon them, in direct relation to the irrigated anatomical area.
\end{abstract}

Keywords: adventitia, arteries, elastic tissue, lamb

\section{INTRODUCTION}

The arterial wall comprises intima, media and adventitia (Miclăuș, 2012). Media is the dynamic tunica of the vessel (Gal and Miclăuș, 2013) and adapts to the internal solicitations, mainly given by blood pressure. Adventitia represents the protective (Aughey and Frye, 2001) and resistant tunica of the vessel and its structure generally adapts to the external solicitations. Adventitia of the elastic arteries is generally thin, while in muscular arteries it is more developed, its thickness being able to equal the media. Structurally, adventitia contains loose connective tissue, in which the fibers have a longitudinal or oblique disposition. Cells are relatively scarce, mainly represented by fibrocytes (Raica et al., 2004). In tunica adventitia of most of the arteries, the elastic fibers are in relatively small quantities. Arteries that contain elastic elements in large quantities were reported in the brain of the human adult (Wolff, 1948). In the intracranian segment of the carotid artery in humans, Masuka et al. (2010) signaled the absence of the external elastic lamina. Fang (1958) and Wilkinson (1972) describe the reduction or even disappearance of the elastic fibers in tunica media and external elastic lamina of the vertebral artery in human. There is little information in the speciality literature on the quantity of elastic tissue in tunica adventitia of arteries.

The aim of this study was to investigate the presence and distribution of the elastic component in large caliber vessels' adventitia in lambs.

\section{MATERIALS AND METHODS}

We harvested samples from 5 hybrid Merino lambs (approximately 3 months old), sacrificed 
by the breeders. Samples were taken from the following arteries: ascending aorta, aortic arch, thoracic and abdominal aorta, internal and external iliac arteries, brachiocephalic trunk, subclavian arteries, bicarotid trunk and carotid arteries. The fragments were histologically processed by paraffin embedding and stained with Verhoeff method.

\section{RESULTS AND DISCUSSION}

Arteries taken into study present a highly different adventitia thickness from one segment to the other, and structurally, this tunica is formed out of collagen and elastic fibers. The proportion between the two types of fibers appears to be different from one vessel to another. In some arteries, adventitia contains a small quantity of elastic fibers (ascending aorta, aortic arch, thoracic aorta, bicarotid trunk, left subclavian artery).

In ascending aorta in lamb, adventitia does not seem to exceed $10 \%$ of the wall thickness and has a relatively loose aspect (Fig. 1). In the pulmonary arterial trunk, the thickness of the tunicae approaches the one in the ascending aorta, excepting tunica adventitia which seems slightly thicker (Fig. 2).

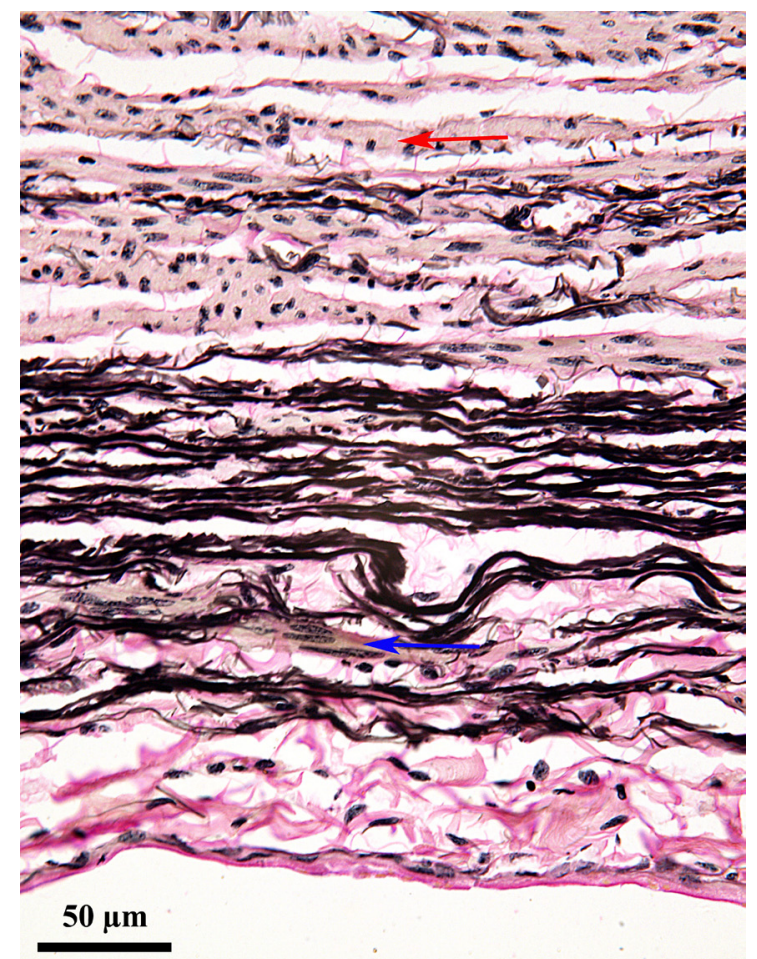

Fig. 1. Ascending aorta: red arrow - media; blue arrow - adventitia (Verhoeff stain)
Adventitia of the aortic arch has a comparable thickness, aspect and structure to the one encountered in the ascending aorta (Fig. 3). In the thoracic descending aorta, the aspect and structure are both comparable to the segments described anteriourly, but the thickness is larger (Fig. 4). In bicarotid trunk there is a discrete increase in the number of elastic fibers present in the adventitia's thickness. The fibers are polymorphic, with a certain distance between them and irregularly distributed, most of them with an oblique or longitudinal arrangement (Fig. 5). In the left subclavian artery, the elastic fibers are present, but relatively rare, with an obvious tendency to dispose as a network (Fig. 6).

Other arteries contain elastic fibers in larger quantities, without exceeding a certain limit (abdominal descending aorta, left and right internal iliac arteries, brachiocephalic trunk, right subclavian artery).

In the abdominal descending aorta, adventitia represents $40 \%$ of the arterial wall thickness and contains relatively fine elastic fibers, well represented numerically and with a highly circular disposition (Fig. 7).

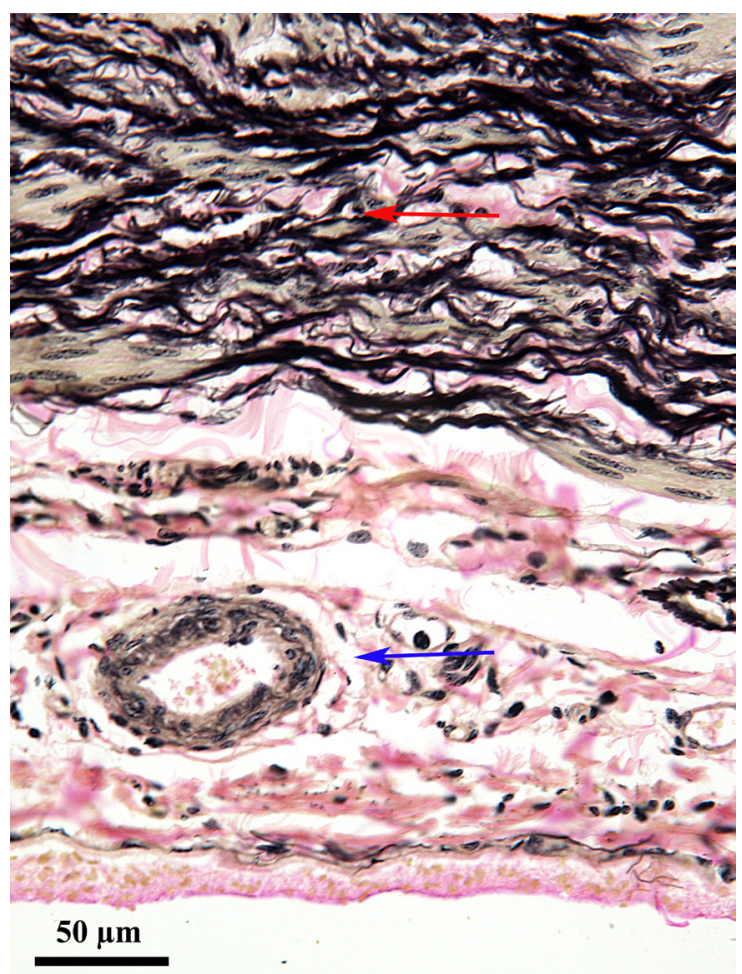

Fig. 2. Pulmonary arterial trunk: red arrow - media; blue arrow - adventitia (Verhoeff stain) 
Adventitia of the internal iliac arteries is thick and contains elastic fibers in all its thickness, but they are finer and significantly lesser in comparison to the ones in the external iliac arteries. The left internal iliac artery contains elastic fibers with a predominantly oblique and longitudinal disposition (Fig. 8) and the right iliac artery contains slightly more elastic fibers, among which most are circularly disposed (Fig. 9). In the case of the brachiocephalic trunk, tunica adventitia occupies approximately $25 \%$ of the wall thickness and the elastic fibers are present in all adventitia's thickness and appear obliquely and longitudinally disposed (Fig. 10).

The right subclavian artery presents approximately the same quantity of elastic fibers as the bicarotid trunk, but here the great majority of fibers are grouped in the internal part of the adventitia (Fig. 11).

There are also vessels in which the elastic component from adventitia is formed out of thick and numerous fibers, markedly exceeding the one from tunica media (left and right external iliac arteries, left and right carotid arteries).

In the external iliac arteries, adventitia is very thick, occupying approximately 45\%

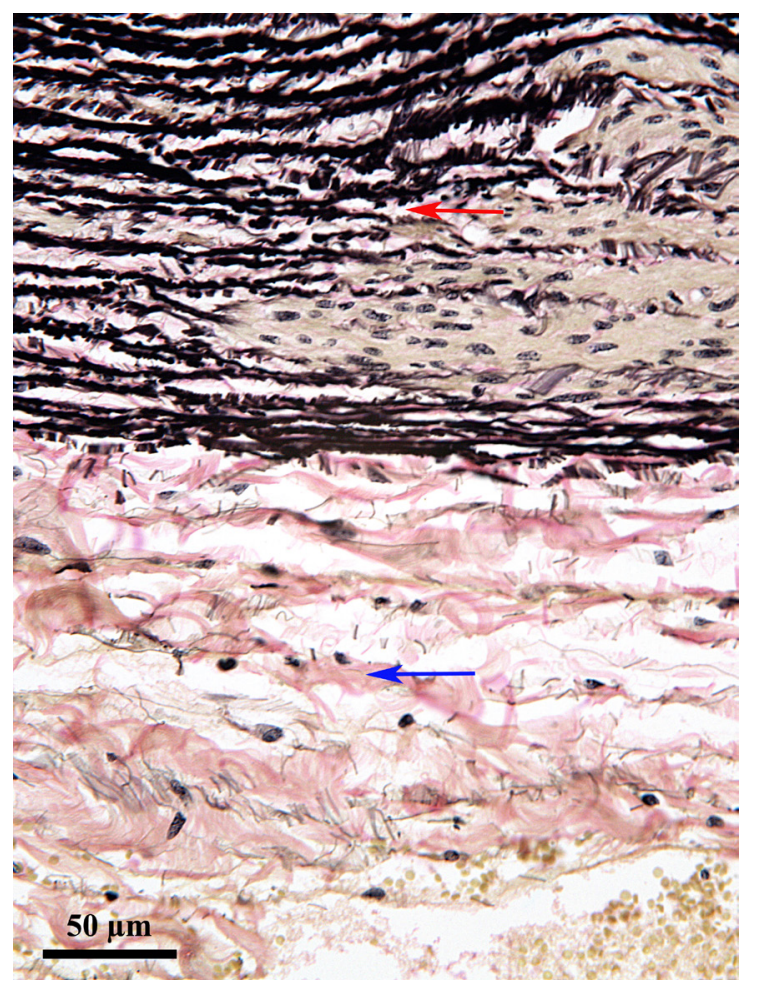

Fig. 3. Aortic arch: red arrow - media; blue arrow adventitia (Verhoeff stain) from the arterial wall thickness. This thick adventitia contains a large quantity of elastic tissue, represented by circularly disposed thick elastic fibers, with a small distance among them, throughout all adventitia's thickness (Fig. 12, 13). In the case of the left carotid artery, adventitia occupies approximately $40 \%$ of the arterial wall thickness and does not present the same structure in all its thickness (Fig. 14). Thus, the external part of the adventitia is formed out of loose connective tissue with very few elastic fibers and in the internal part, the elastic component is very well represented (the proportion between the collagenic and elastic component is approximately 1:1). The elastic fibers from adventitia, besides the fact that they are numerous, appear to be fairly thick, many of them approaching the thickness of the elastic lamellae in tunica media. Regarding their disposition, the majority are circularly disposed, with a small distance between them (they are fairly dense). The right carotid artery resembles the left one, but in this artery, the area occupied by elastic tissue is slightly thicker (Fig. 15).

Among the arteries taken into study there are larger or smaller differences from one segment

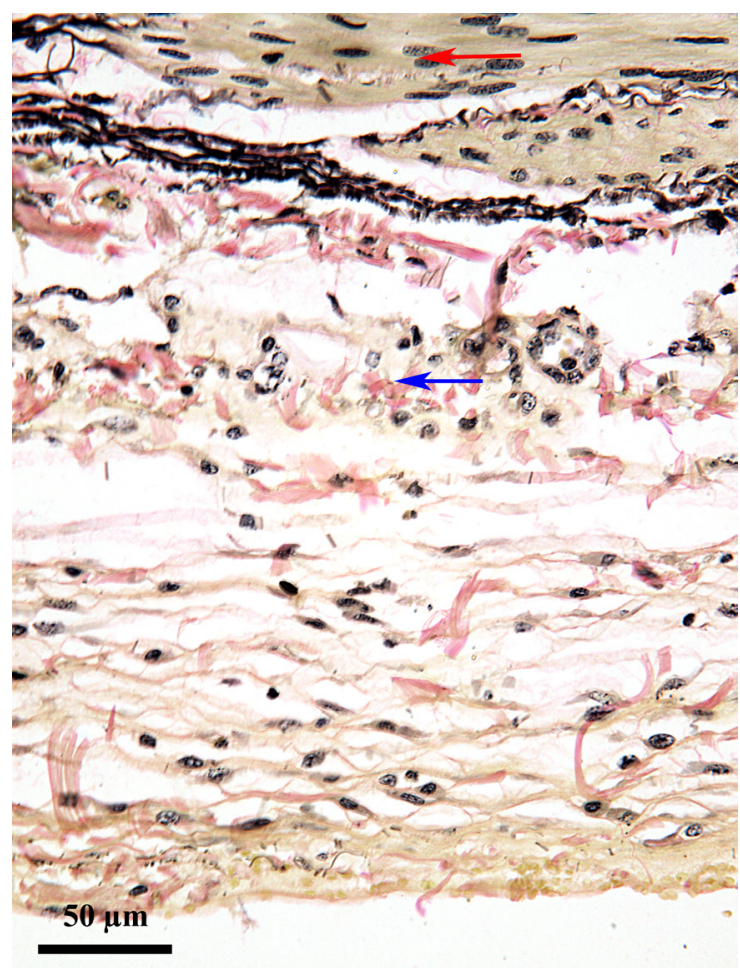

Fig. 4. Thoracic descending aorta: red arrow - media; blue arrow - adventitia (Verhoeff stain) 


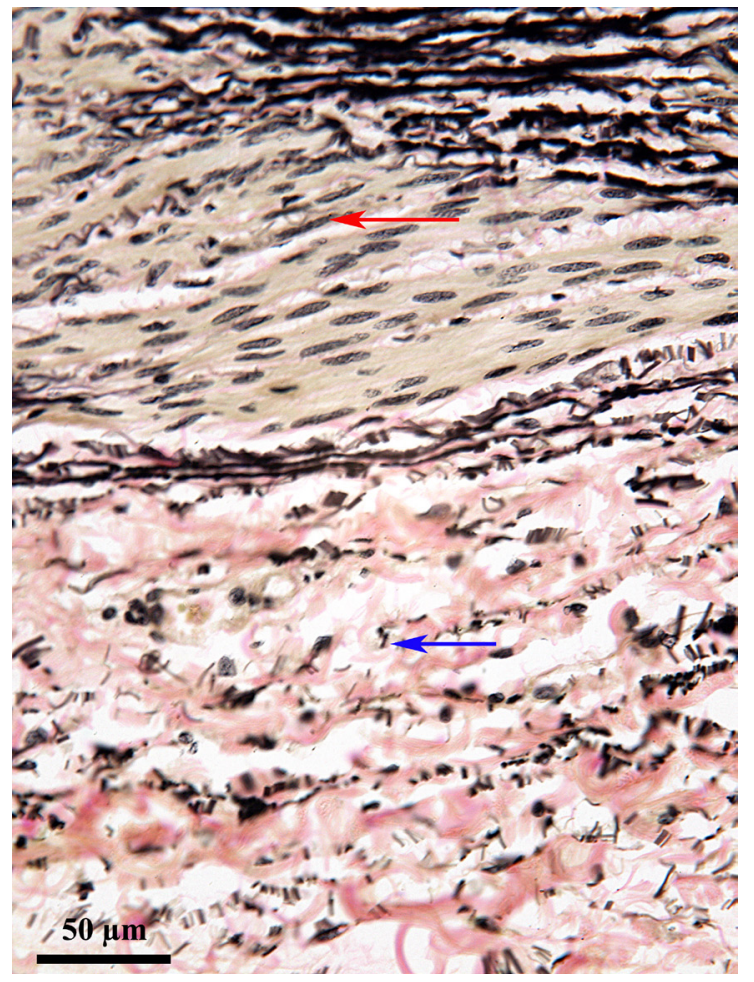

Fig. 5. Bicarotid trunk: red arrow - media; blue arrow - adventitia (Verhoeff stain)

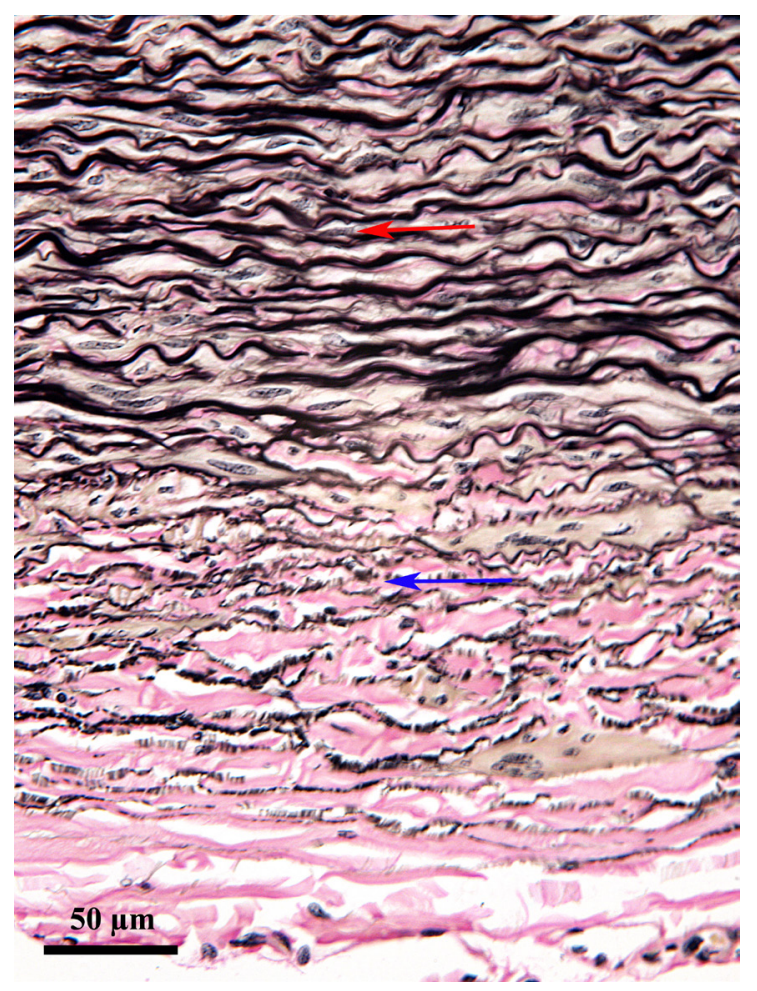

Fig. 7. Abdominal descending aorta: red arrow media; blue arrow - adventitia (Verhoeff stain)

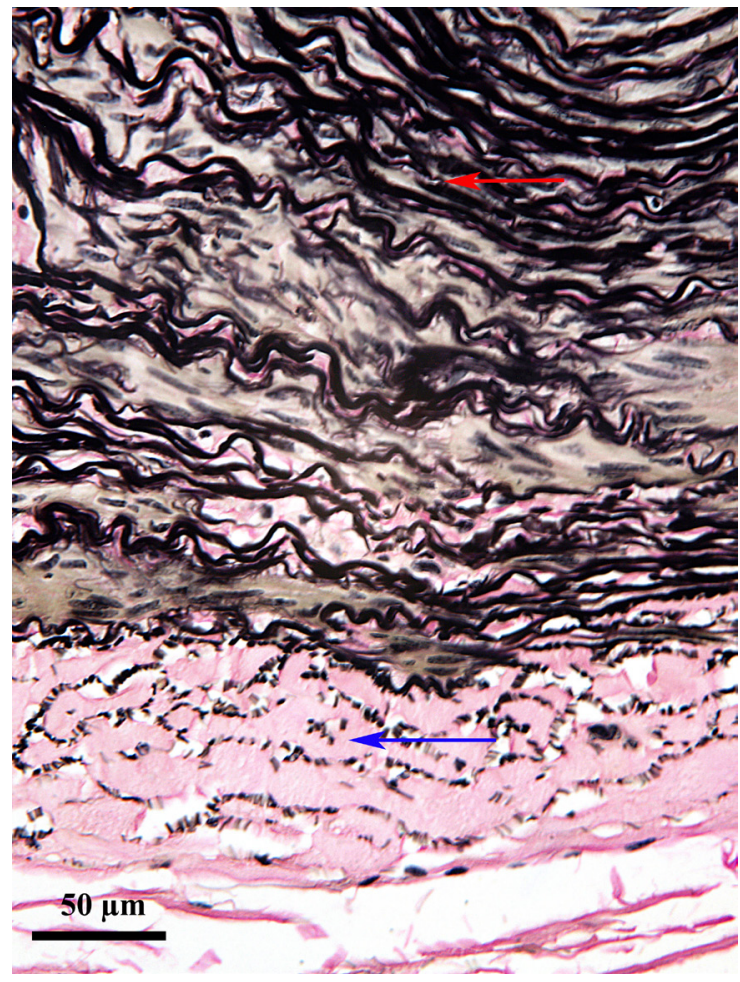

Fig. 6. Left subclavian artery: red arrow - media; blue arrow - adventitia (Verhoeff stain)

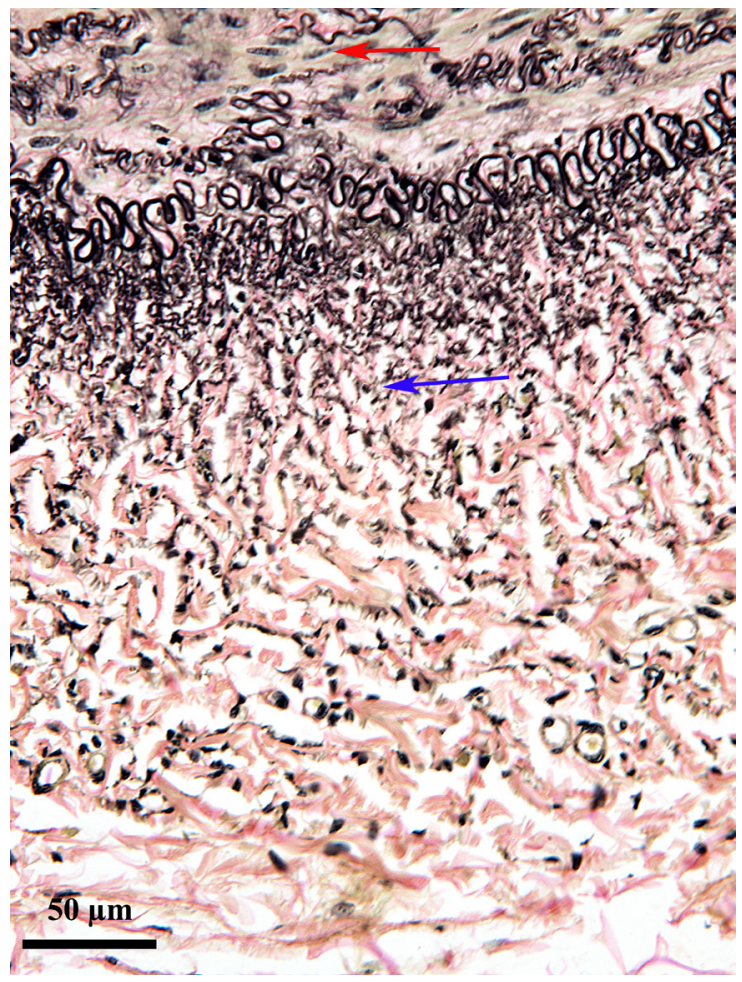

Fig. 8. Left internal iliac artery: red arrow - media; blue arrow - adventitia (Verhoeff stain) 


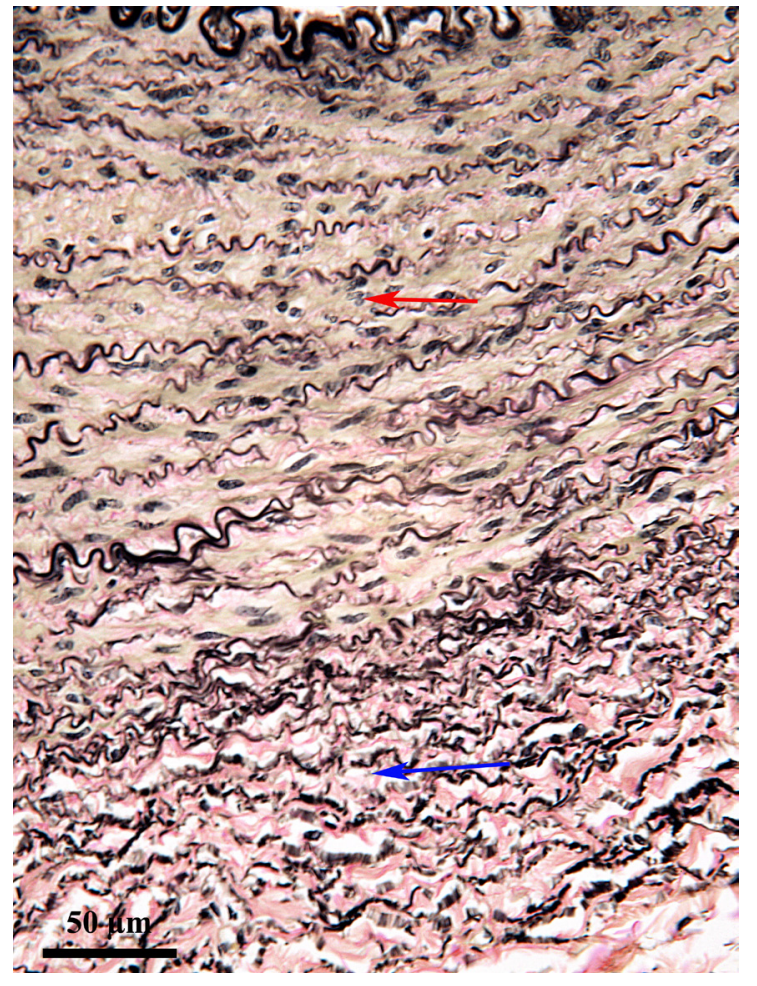

Fig. 9. Right internal iliac artery: red arrow - media; blue arrow - adventitia (Verhoeff stain)

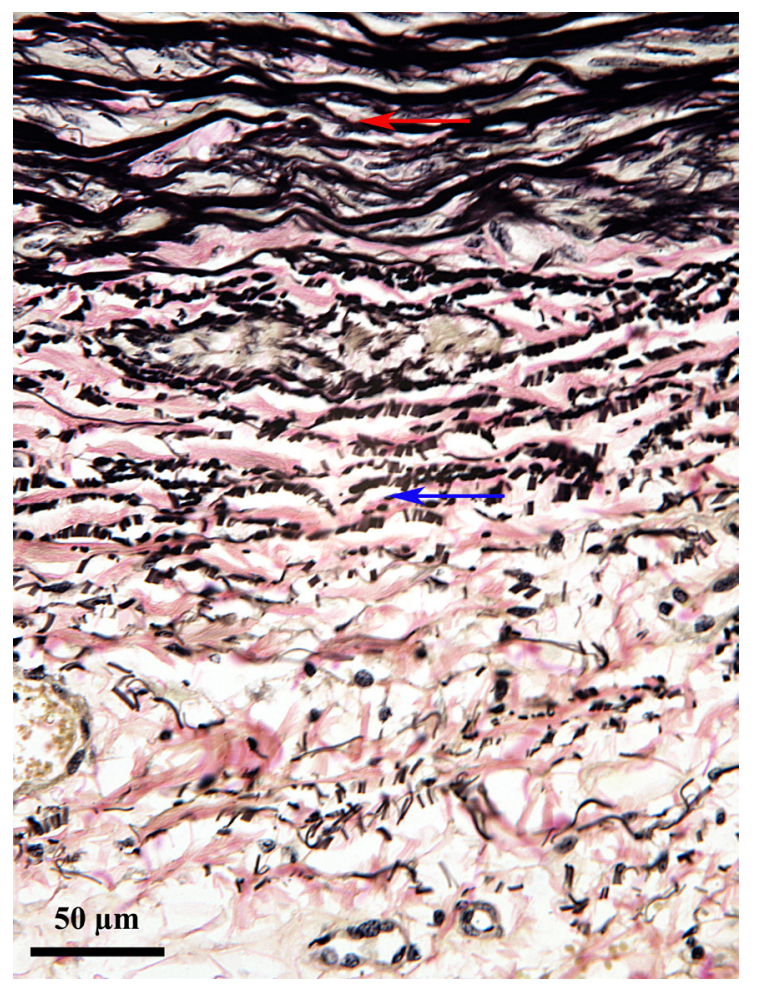

Fig. 11. Right subclavian artery: red arrow - media; blue arrow - adventitia (Verhoeff stain)

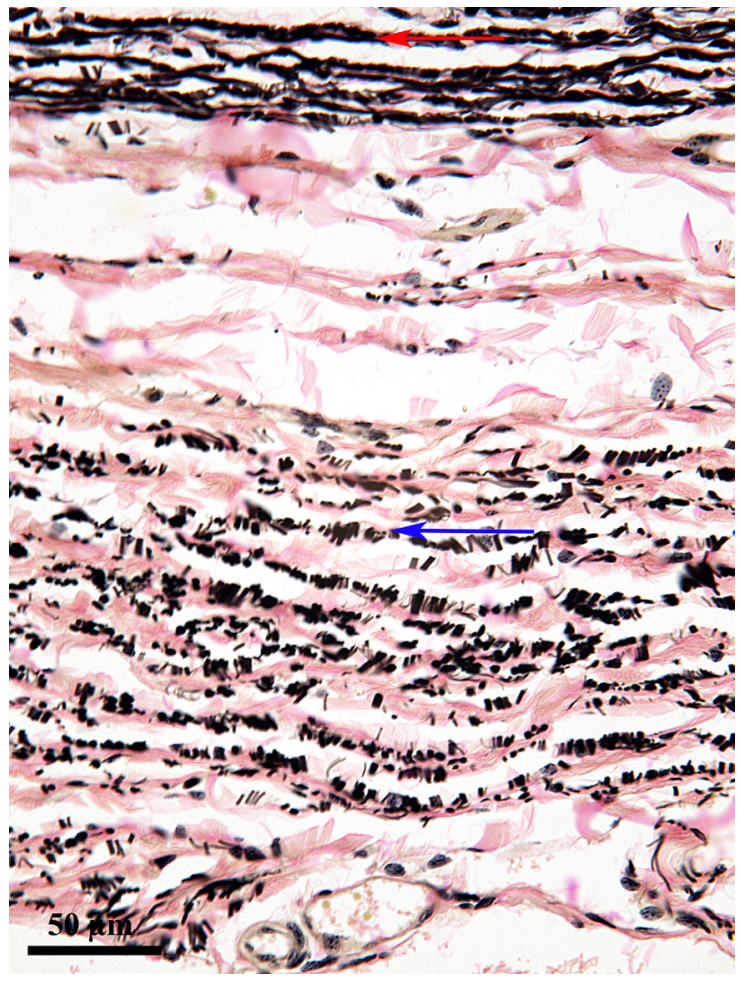

Fig. 10. Brachiocephalic trunk: red arrow - media; blue arrow - adventitia (Verhoeff stain)

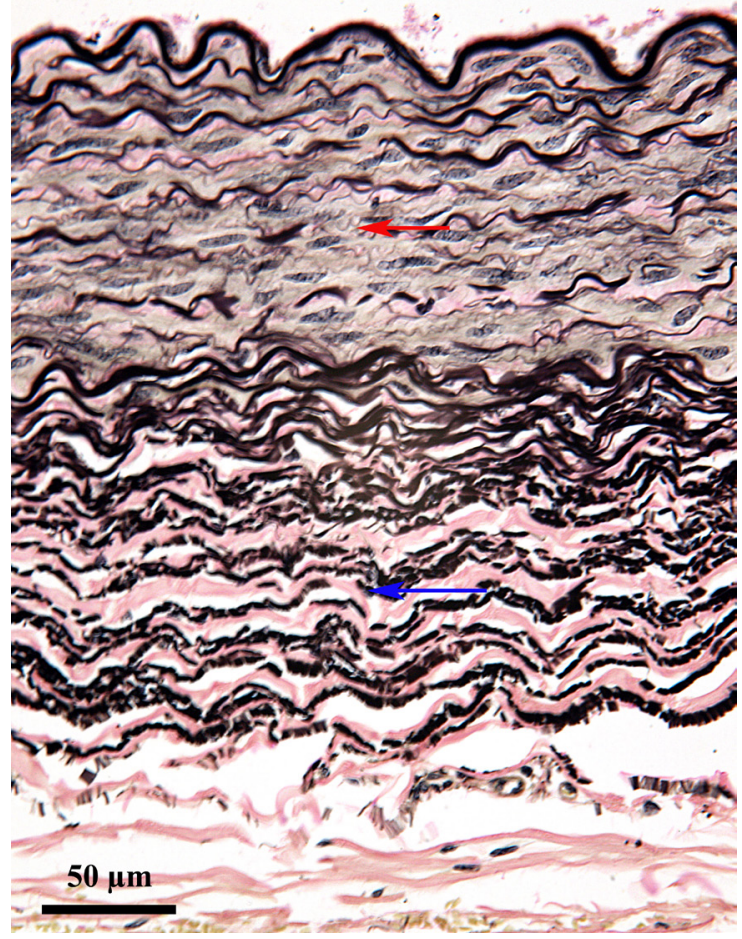

Fig. 12. Left external iliac artery: red arrow - media; blue arrow - adventitia (Verhoeff stain) 
to another, regarding the thickness of tunica adventitia. These differences are not due to the caliber of each arterial segment studied, but are given by the position and distance in which each segment lies in relation to the heart and also depending on the irrigated anatomical area. Regarding the structure, adventitia is formed out of collagen and elastic fibers, and the proportion between these two is presented in the speciality literature as being in the favor of the collagen ones. Our study highlighted the fact that in lamb, there are differences regarding the proportion between the two components.

Thus, in the case of some of the arteries, the proportion between the two components is highly in the favour of collagen: ascending aorta, aortic arch, thoracic descending aorta, bicarotid trunk and left subclavian artery. There is no correlation between tunica adventitia's thickness and the proportion between the two basic components in arteries metioned above. The ascending aorta has the thinnest adventitia, while in the thoracic descending aorta and bicarotid trunk it appears several times thicker. Nontheless, the proportion between the two structural components is not necessarily identical, but at a comparable level,

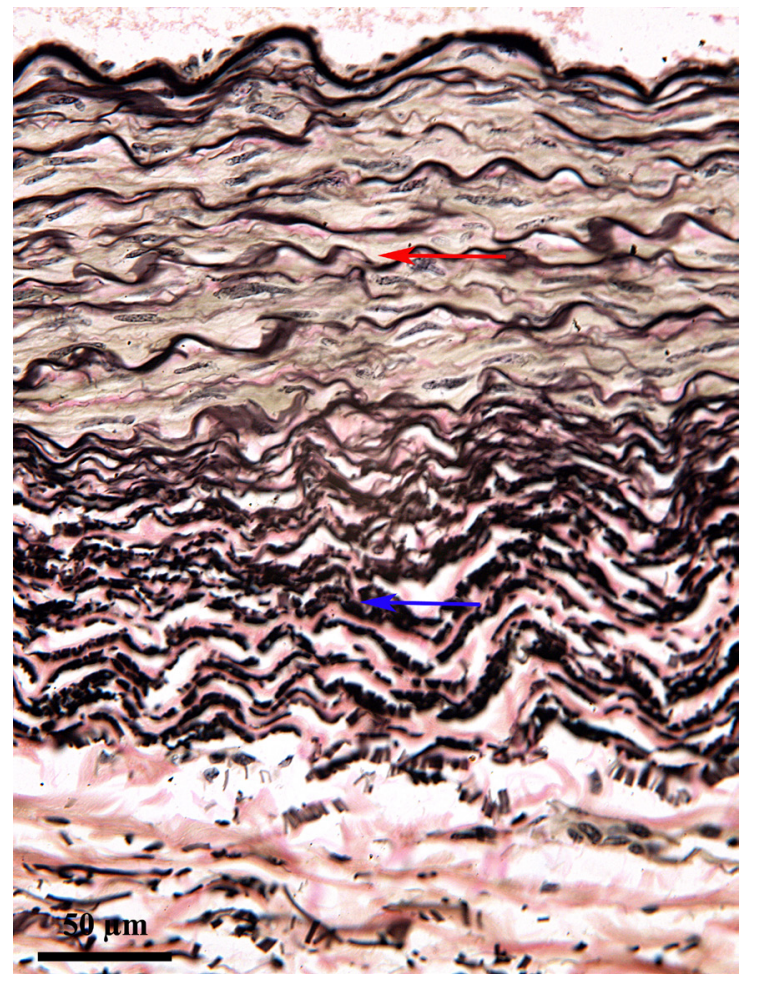

Fig. 13. Right external iliac artery: red arrow - media; blue arrow - adventitia (Verhoeff stain) tunica adventitia of all these vessels containing a small quantity of elastic tissue. This aspect reveals the fact that the thickness of adventitia is not necessarily an element that decisively influences the proportion between the collagenic and elastic components.

In the case of other arteries, an increase of the elastic tissue quantity from tunica adventitia is observed, but not to such an extent that it approaches closely the collagenic component or equals it. The descending abdominal aorta, the left and right internal iliac arteries, brachiocephalic trunk and right subclavian artery are in this situation. There are also differences in adventitia thickness of the listed vessels, but a direct connection between adventitia and elastic tissue quantity in its structure is not noticed here either. The quantity of elastic tissue in tunica adventitia of these arteries differs between certain limits from one arterial segment to another, but the differences are not necessarily significant. We mention that the disposition of the elastic fibers in adventitia of these arteries is not closely rigurous, but the fibers a practically oriented in many directions. Their thickness varies from fine (the majority) to a medium one.

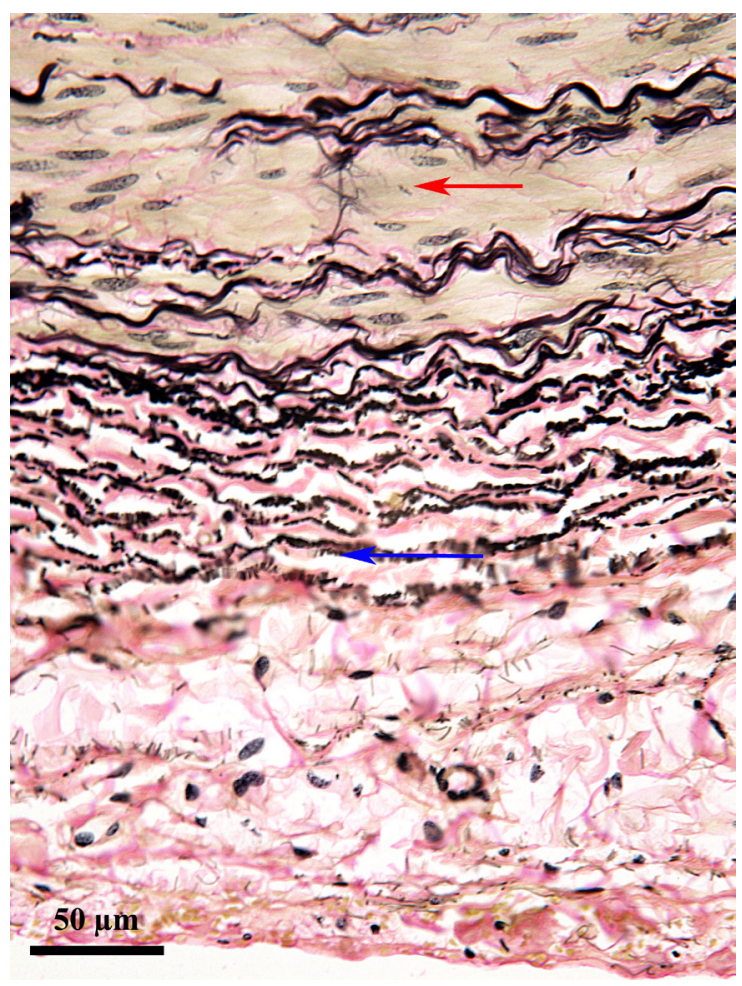

Fig. 14. Left carotid artery: red arrow - media; blue arrow - adventitia (Verhoeff stain) 
The third category is represented by arteries whose adventitia contains a very well represented adventitia. The arteries that fall under this category are the external iliac (left and right) and carotid arteries (left and right). The quantity of elastic tissue from tunica adventitia of these vessels is so large that in some of them (left external iliac artery) the proportion between the elastic and collagenic component tends to be very close. Moreover, the elastic fibers in adventitia of these arteries are significantly thicker in comparison to the arterial segments previously presented and have a relatively rigurous disposition. Thus, in these arteries we have thick and sinuous elastic fibers, with a lamellar aspect and most of them circularly disposed. By their thickness, aspect and orientation, the elastic fibers in adventitia of these arteries, the general aspect tends to be comparable to the one in the media of elastic arteries. Considering the fact that in tunica media of these arteries, the elastic component is obviously less well represented than in adventitia, we can affirm that in these arteries the elasticity of the wall is mainly given by adventitia and secondly by media. The situation seems slightly paradoxical if we do not take into account de anatomical area irrigated by these arteries. These areas are far more solicitated mechanically, with a larger mobility of the surrounding components in comparison to the previously presented arteries. In this context, the thickness, aspect and orientation of the elastic fibers in arteries from this category appear as an

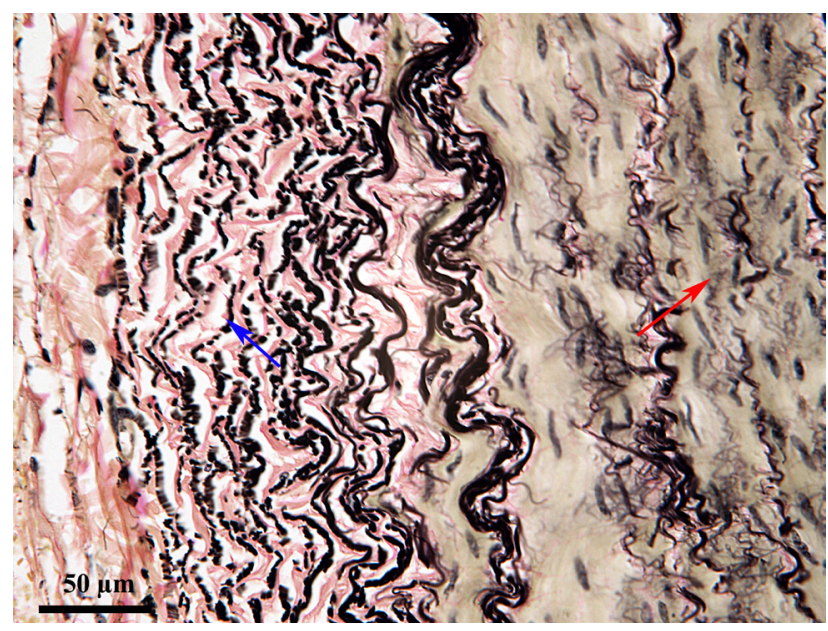

Fig. 15. Right carotid artery: red arrow - media; blue arrow - adventitia (Verhoeff stain) adaptation to the external solicitations exerting upon them, in a direct relation to the irrigated anatomical area.

\section{CONCLUSION}

Tunica adventitia, in the studied large caliber arteries, presents differences in thickness depending on the caliber and irrigated area and also differences regarding the proportion between the structural components and their disposition.

The proportion between the collagenic and elastic components is different in adventitia of the studied arteries, the quantity of elastic tissue being smaller in some, well represented in others and very well represented in the rest of them.

Iliac and carotid arteries contain significantly more elastic tissue in adventitia than in media, and is disposed as concentric lamellae which follow the aspect encountered in tunica media of the typical elastic arteries.

The relatively large differences regarding the quantity and disposition of the elastic tissue in tunica adventitia of the studied vessels do not seem to have a direct connection with its thickness, but appear as an adaptation to the external solicitations exerted upon them, which are a lot higher in some anatomical areas than others.

\section{REFERENCES}

1. Aughey E, Frye F L (2001). Comparative Veterinary Histology with Clinical Correlates, State University Press, Iowa , 71-81.

2. Fang $H$ (1958). A comparison of blood vessels of brain and peripheral blood vessels. In Wright IS, Millikan CH. Cerebral Vascular Diseases, Grune \& Stratton, New York, 17-22.

3. Gal A F, Miclăuș V (2013). Histology. Risoprint, ClujNapoca, 165-180.

4. Masuoka T, Hayashi N, Hori E, Kuwayama N, Ohtani O, Endo S (2010). Distribution of internal elastic lamina and external elastic lamina in the internal carotid artery: possible relationship with atherosclerosis. Neurol Med Chir (Tokyo) 50(3):179-82.

5. Miclăuș V (2012). Histologie specială și embiologie generală. Ed a II-a, Risoprint, Cluj-Napoca,7-22.

6. Raica M, Mederle O, Caruntu ID, Pintea A, Chindris AM (2004). Histologie teoretica și practică. Brumar, Timișoara, 101-120.

7. Wilkinson IMS (1972). The vertebral artery. Extracranial and intra-cranial structure. Arch Neurol 27: 392-396.

8. WolfT HG (1948). Headache and Other Head Pain. Oxford Univ Press, New York. 